[Supplementary materials to accompany]

\title{
Directed-Assembly of Transition-Metal-Coordinated Molecular Loops and Squares from Salen-Type Components. Examples of Metallation-Controlled Structural Conversion
}

\author{
Shih-Sheng Sun, Charlotte L. Stern, SonBinh T. Nguyen,** and Joseph T. Hupp* \\ Department of Chemistry, Center for Nanofabrication and Molecular Self-Assembly, and \\ Institute for Environmental Catalysis \\ Northwestern University \\ 2145 Sheridan Road \\ Evanston, IL 60208 \\ Fax: (847) 491-7713 \\ E-mail:stn@chem.northwestern.edu \\ jthupp@chem.northwestern.edu
}

Supporting Information Available: Crystallographic data in CIF format, NMR and MS spectra for compounds 12-18. This material is available free of charge via the Internet at http://pubs.acs.org. 


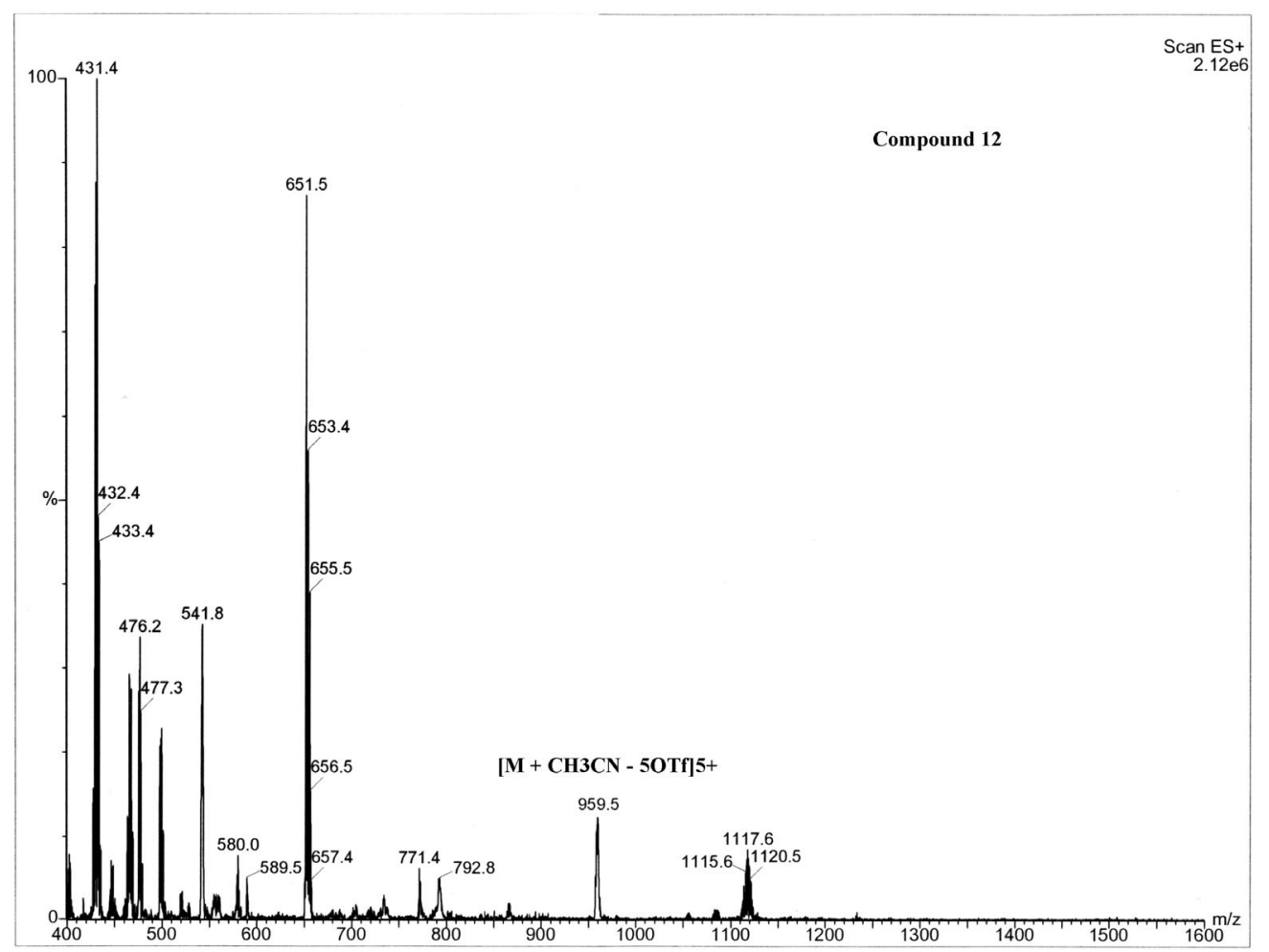

F19 OBSERVE
STANOARD PARAMETERS

Pulse sequence: s2pul

Solvent: Acetone
Antient : temperat

Mercury-4008B " mercury $400 "$

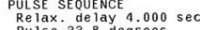

Aca. time 0.600 sec

Aca time 0.600
witan $50000.0 \mathrm{~Hz}$

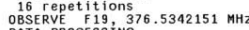

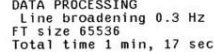

Compoun 12, F-19 spectrum

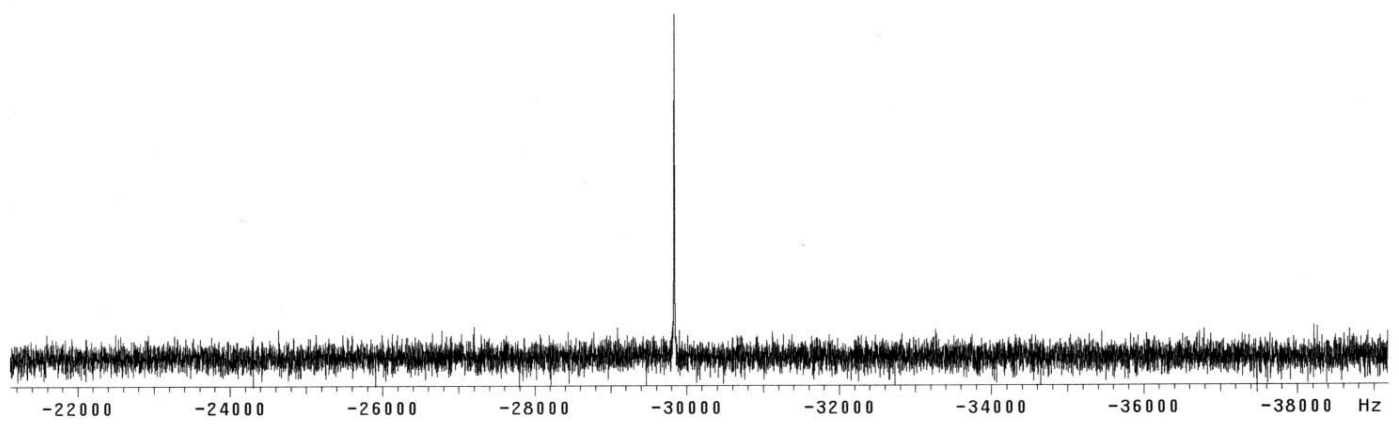



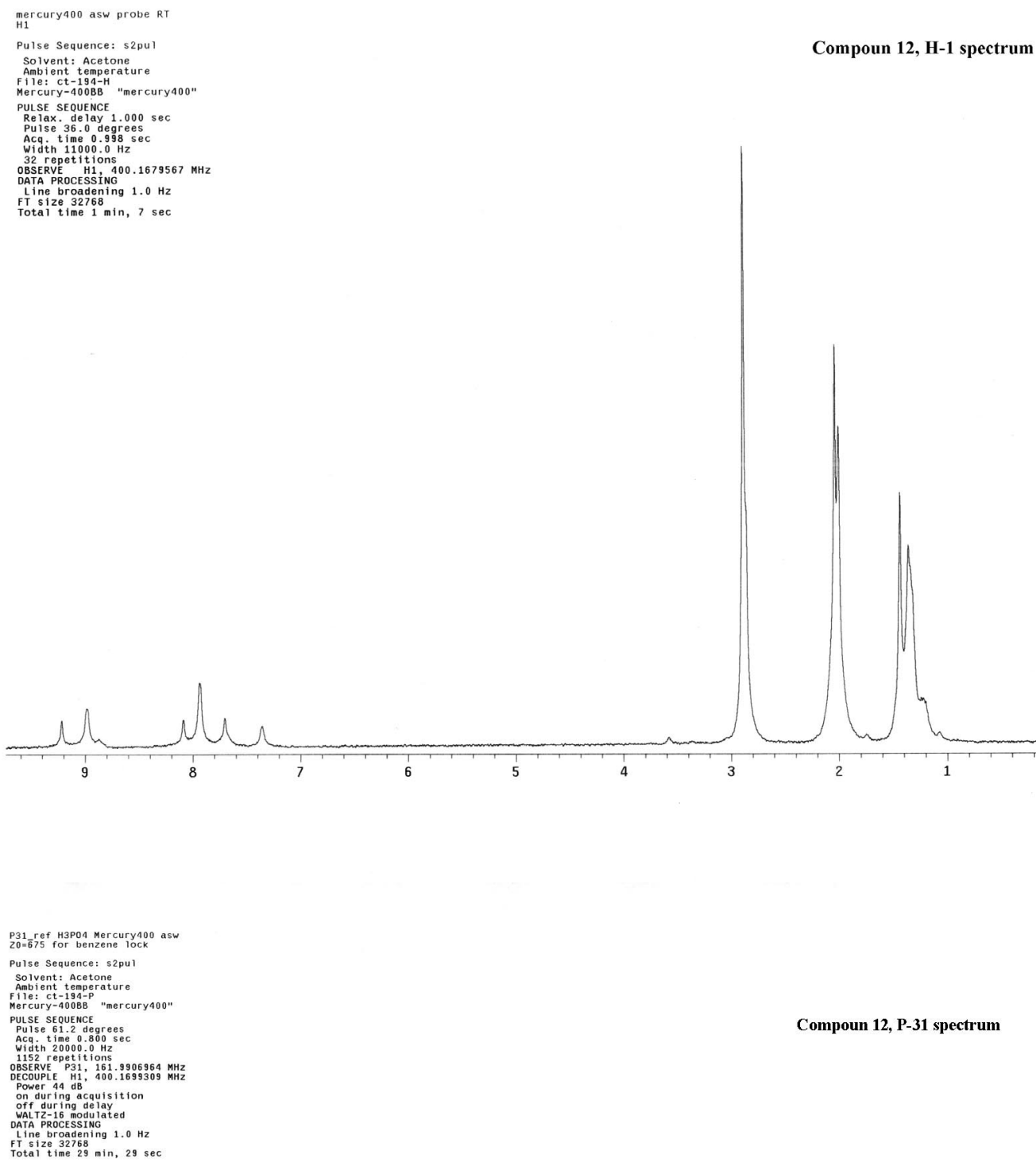

Compoun 12, P-31 spectrum
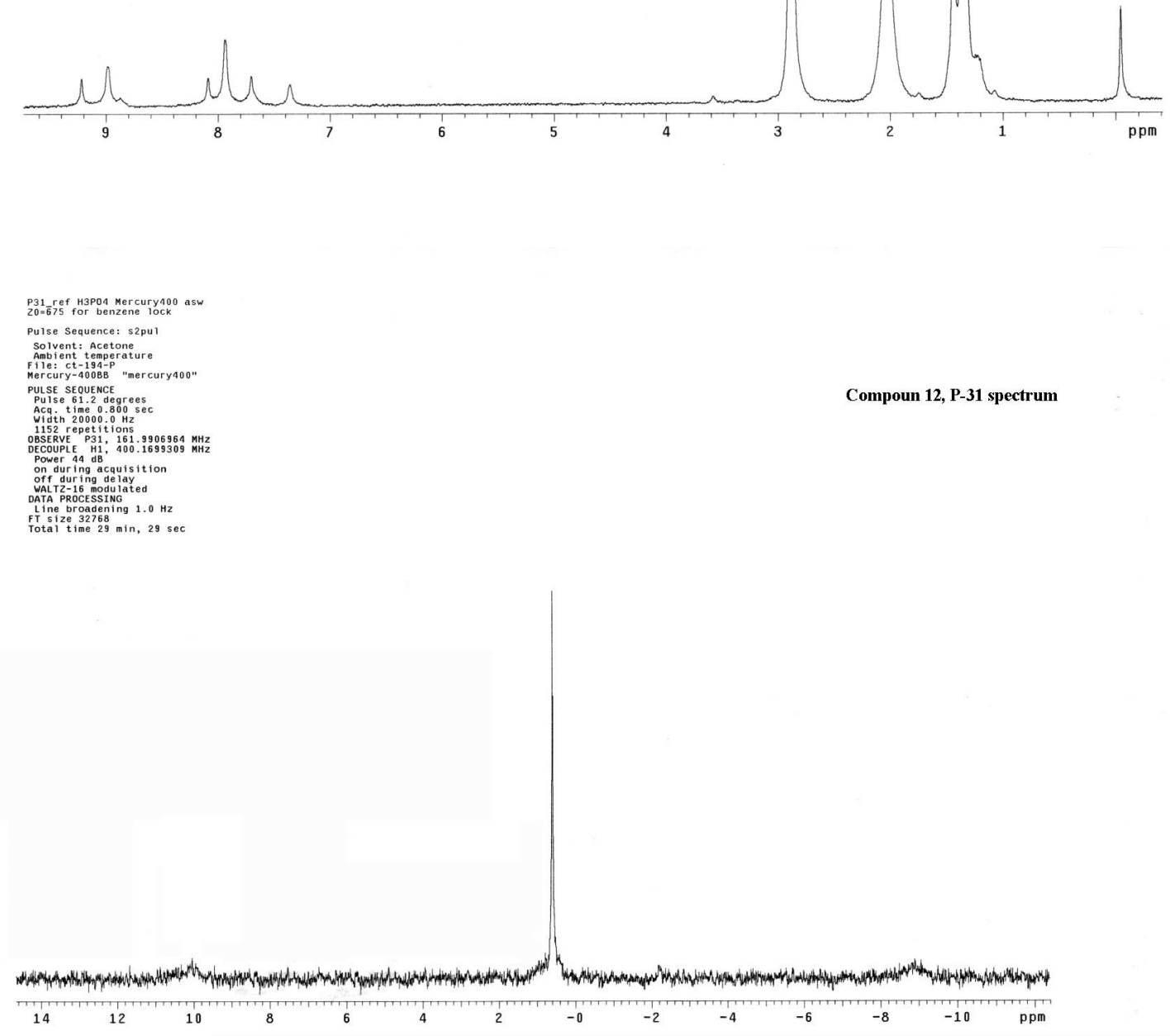


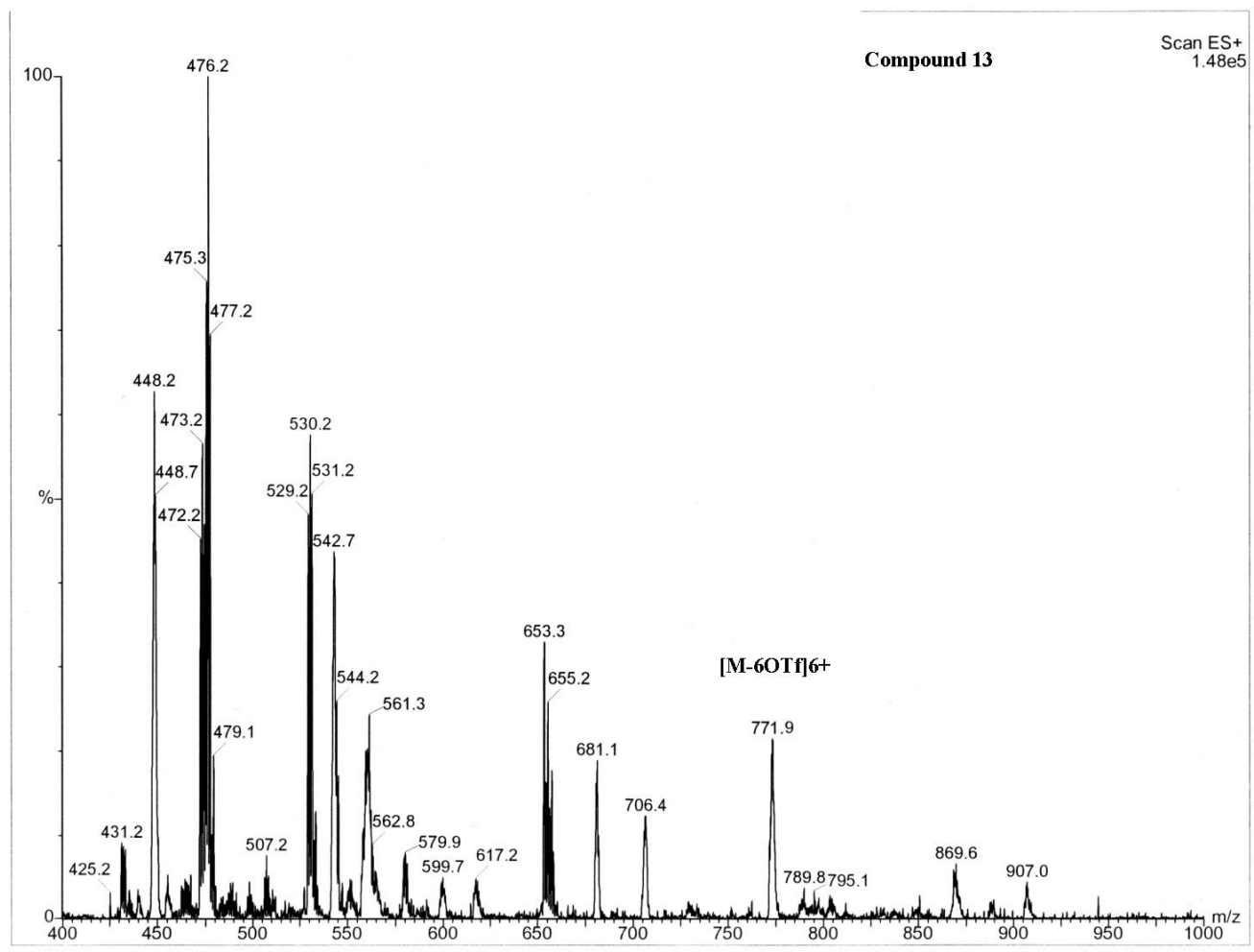

F19 OBSERVE
STANDARD PARAMETERS

Pulse Sequence: s2pu

Solvent: Acetone
Ambient temperat

Mercury-400BB

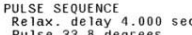

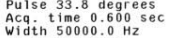

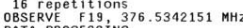

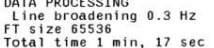

Compoun 13, F-19 spectrum

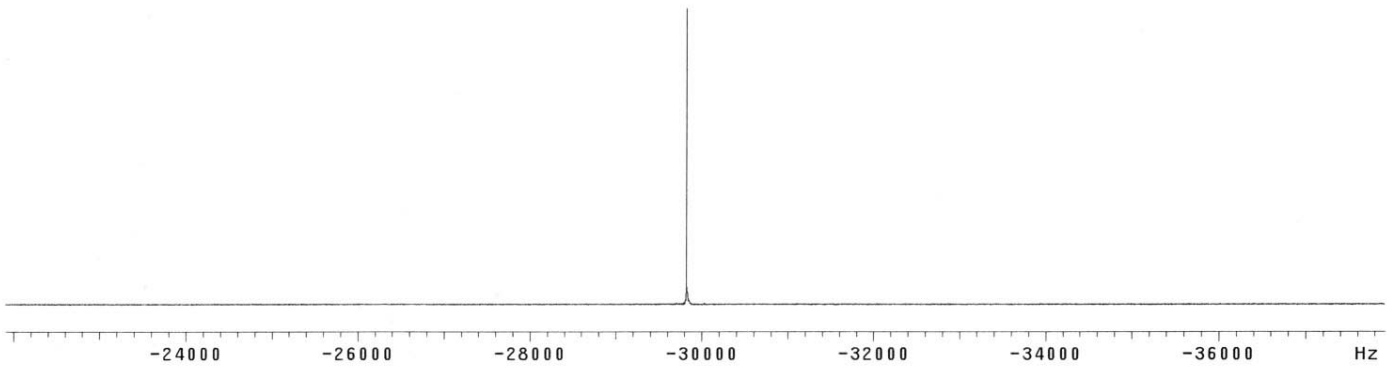




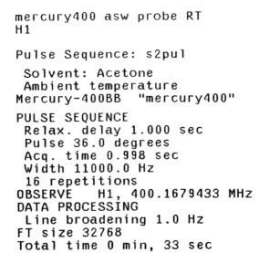

Solvent: Acetone
Ambient temperature

UULSE SEQUENCE

Pulse 36.0 degrees
Acq.

16 repetitions
OBSERVE
OB
DATA PROCEST

FT size 32768 min, $33 \mathrm{sec}$
Total time $0 \mathrm{~min}$

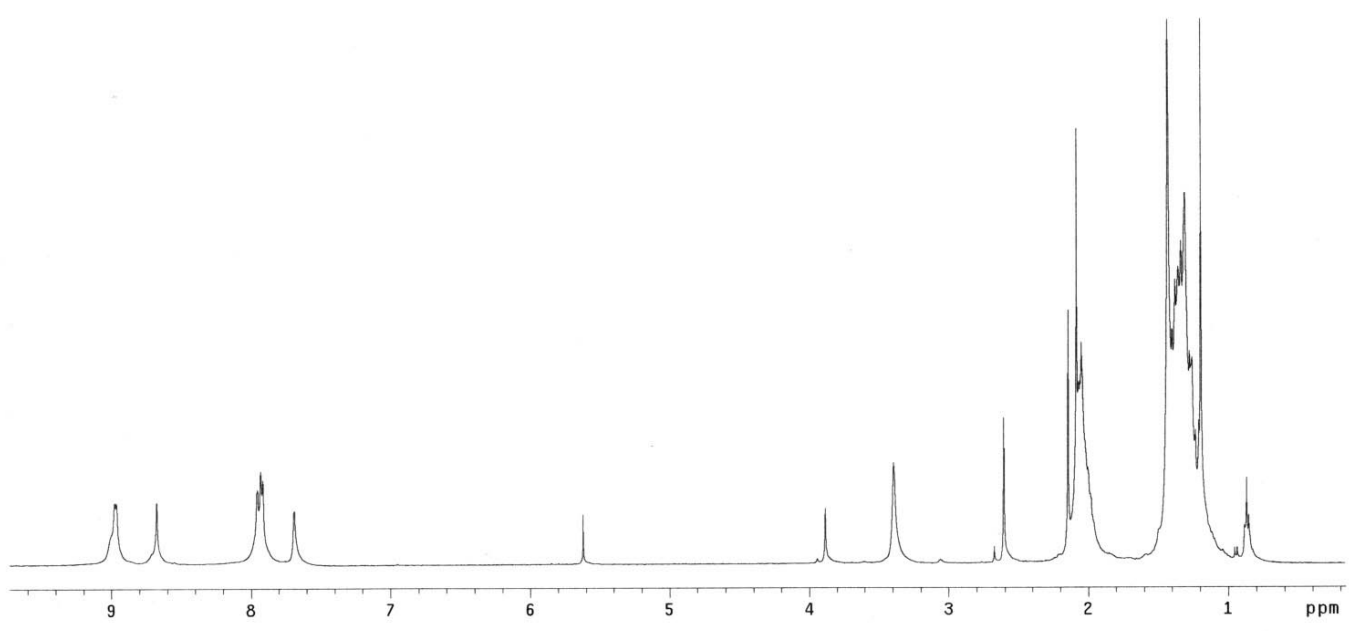

P31 ref H3PO4 Mercury400 asw
$20=\overline{6} 75$ for benzene lock

Pulse Sequence: s2pul

Solvent: Acetone
Amblent temperature
file: ct-211-p

Amb ent temperature
file: ct-21-p
Hercury-400BB "mercury400"

PULSE SEDUENCE
PuIse 61.2 degrees

Acq
Widtime
Wide $20000.00 \mathrm{~Hz}$

256 repetitions
OBSERE P31 161.9906818 MHZ
DECOUPLE H1, 400.169909 MHZ

Power $44 \mathrm{~dB}$
on during acquisition

off during de lay
off dut
WAT -16 modulated

DAA PROCESSING $1.0 \mathrm{~Hz}$

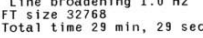

Compoun 13, P-31 spectrum

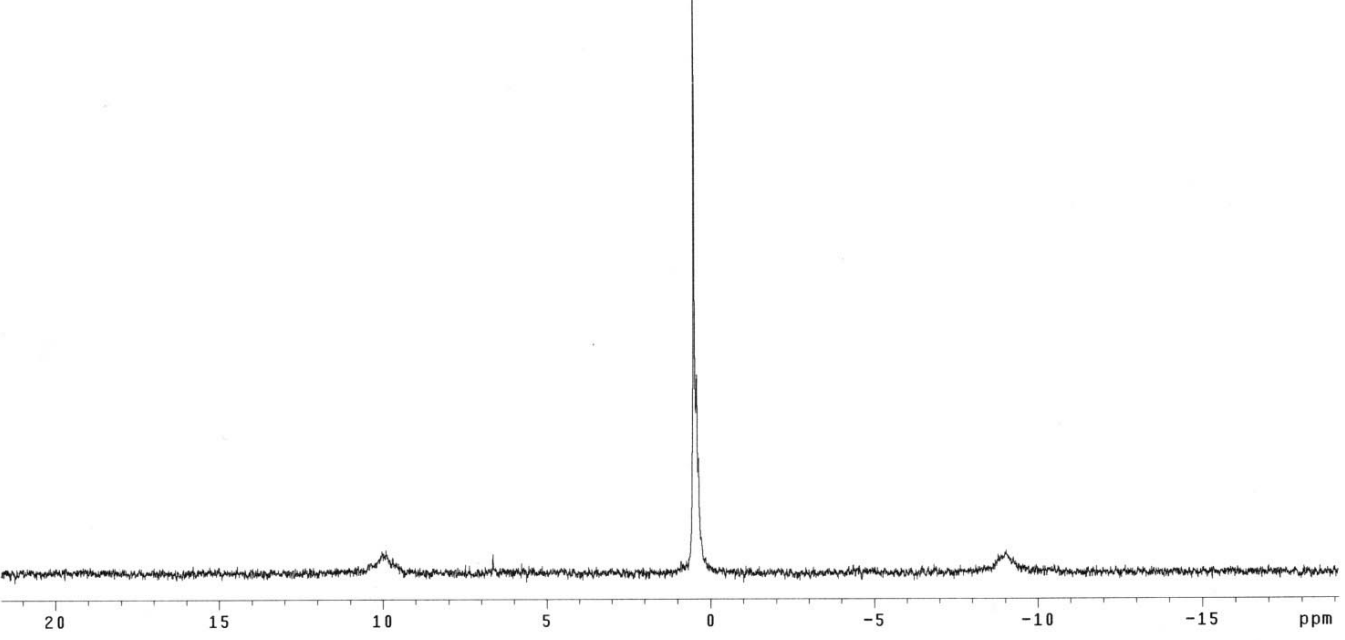


F19 OBSERVE
STANDARD PARAMETERS

Pulse Sequence: s2pul

Solvent: CD3CN
Ambient temperature
mercury-400BB "mercury400"

PULSE SEDUENCE

Pulse 33.8 degrees

Acg time $0.600 \mathrm{sec}$
Width $5000.0 \mathrm{~Hz}$

16 repetitions
OBSERVE F19. $376.5435331 \mathrm{MHZ}$

DATA PROCESSING
Dine broadening $0.3 \mathrm{~Hz}$

Line broadening $0.3 \mathrm{~Hz}$
FT size $65536 \mathrm{~min}, 17 \mathrm{sec}$
Total time $1 \mathrm{~min}, 17 \mathrm{se}$

Compoun 14, F-19 spectrum
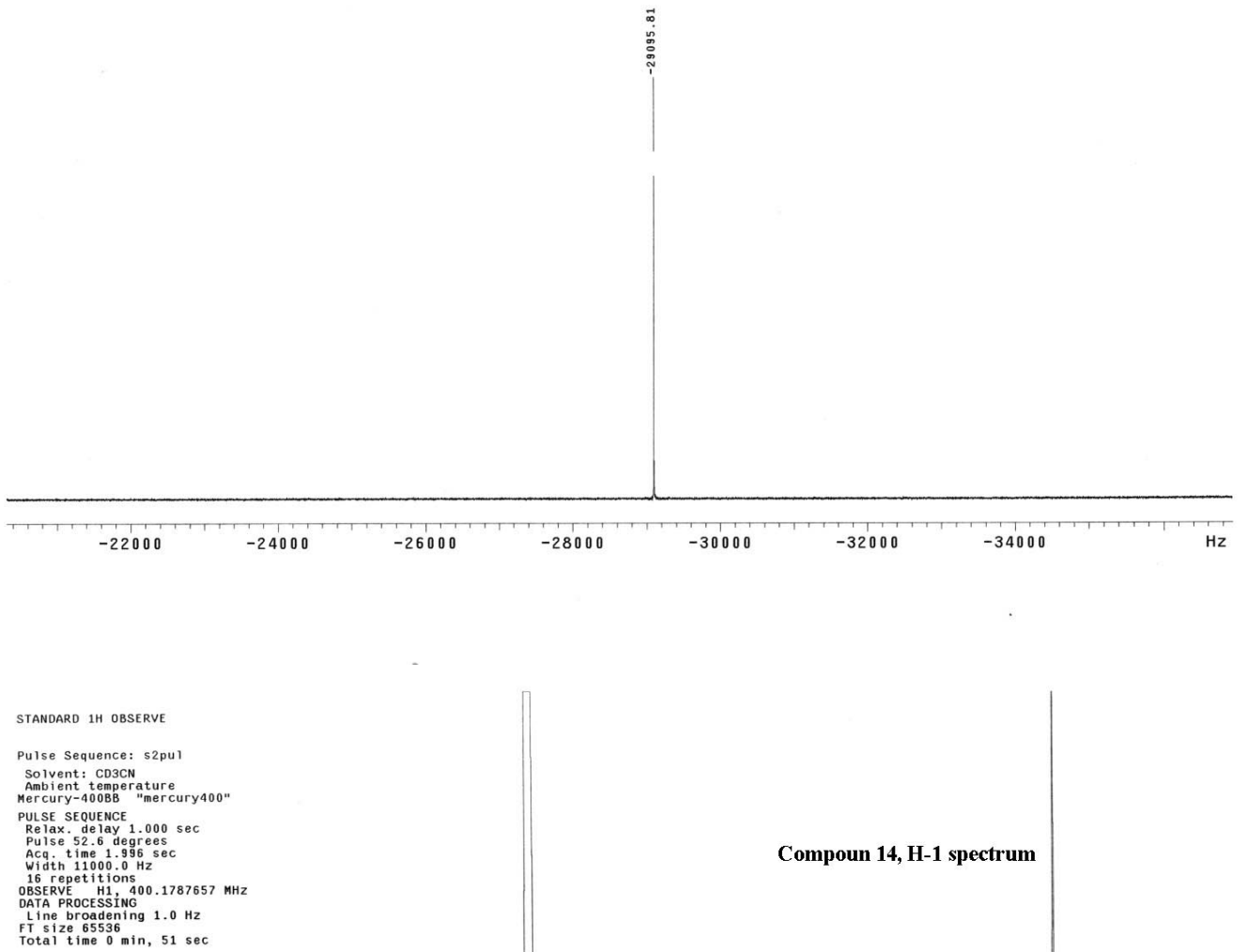

Total time 0 min, 51 se

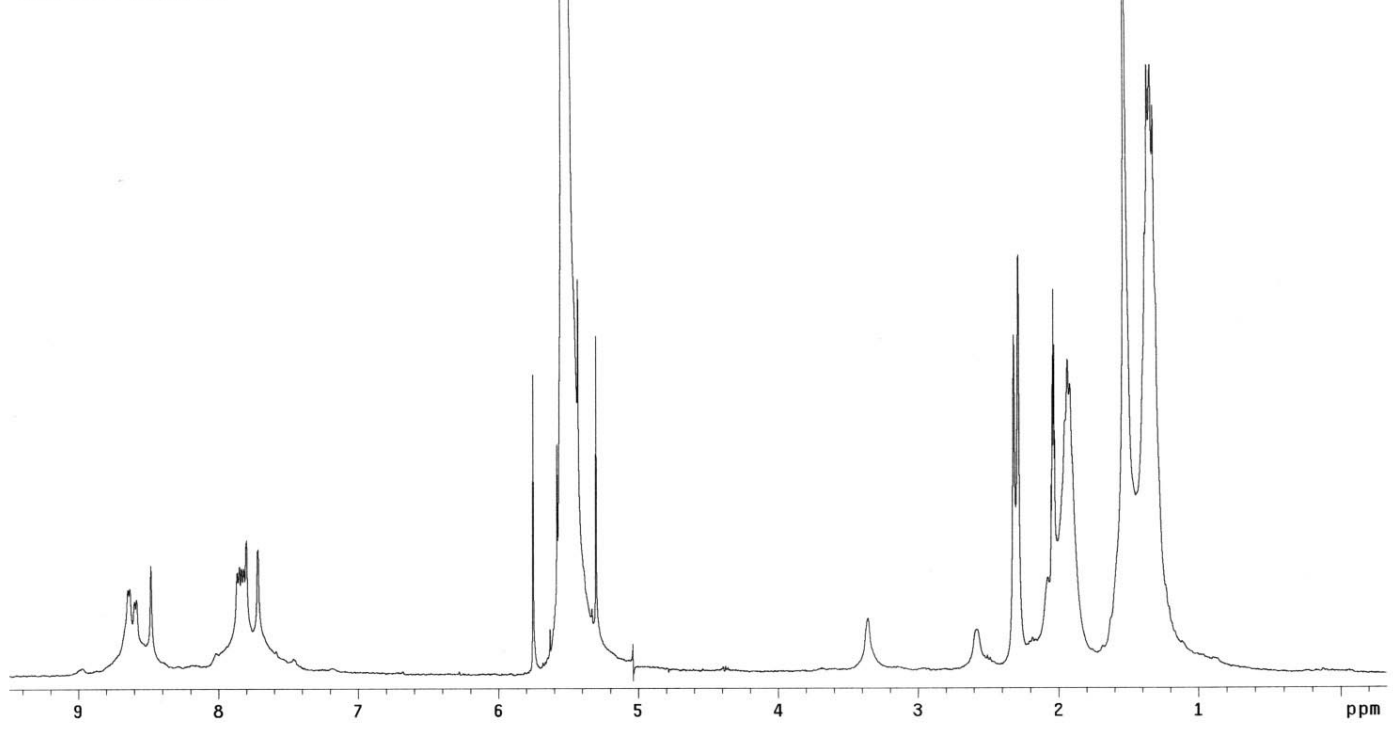




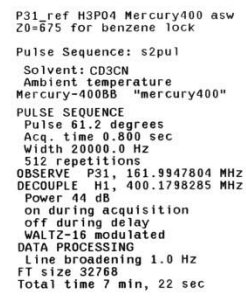

Compoun 14, P-31 spectrum
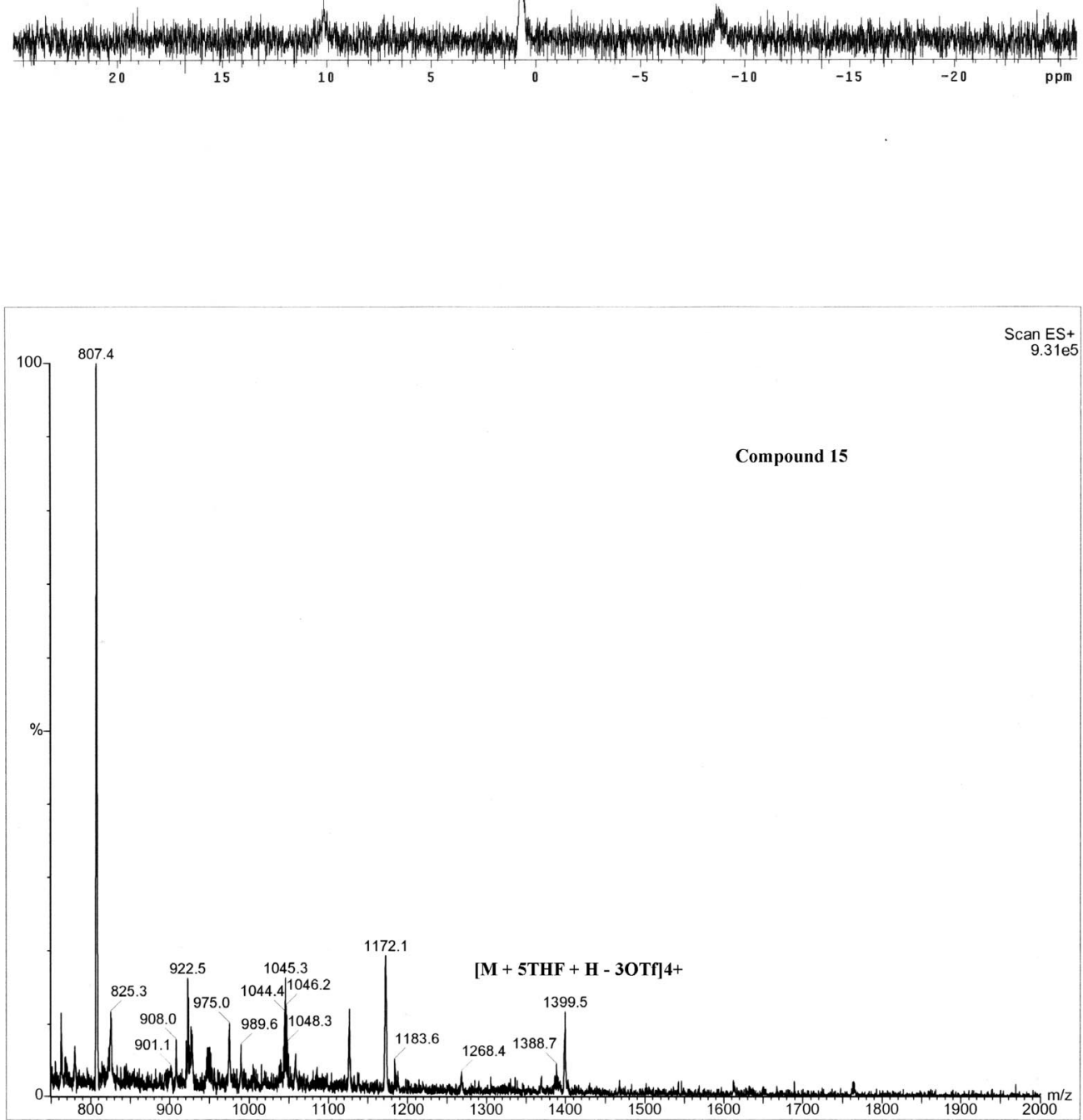
F19 OBSERVE
STANOARD PARAMETERS

Pulse Sequence: s2pul

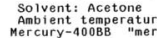

PULSE SEQUENCE

pulse 33 a 4.000 se

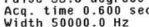

16 repetitions
OBSERVE F F $19.376 .5342151 \mathrm{MHZ}$

DAIA PROCESSING
Line broadening $0.3 \mathrm{~Hz}$

FT $512 e 6556$
Total time $1 \mathrm{~min}, 17 \mathrm{sec}$

Compoun 15, F-19 spectrum

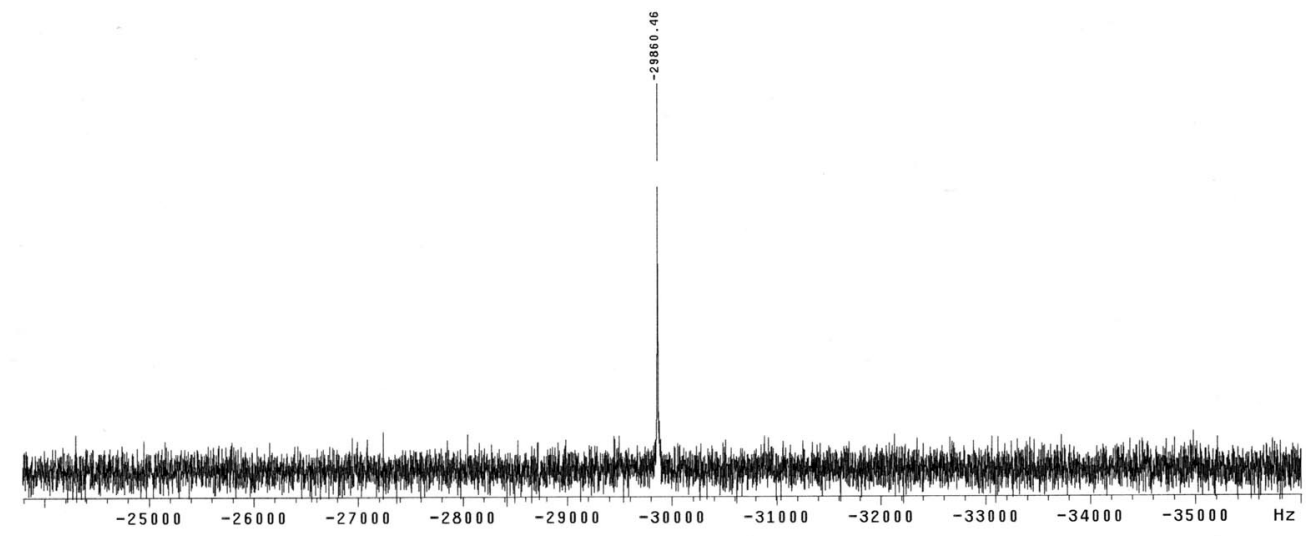

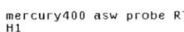

Pulse Sequence: s2pul

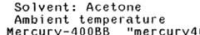

PULSE SEQUENCE

Relax de lay $1.000 \mathrm{sec}$
Pulse 36.0 deerges

Acq
Ant ime 0.998 sec
Width 11000

Width repet titins
OBSERVE H1, $400.1679420 \mathrm{MHz}$

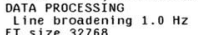

FT Size 32768 in, $7 \mathrm{sec}$
Total time $1 \mathrm{~min}$.

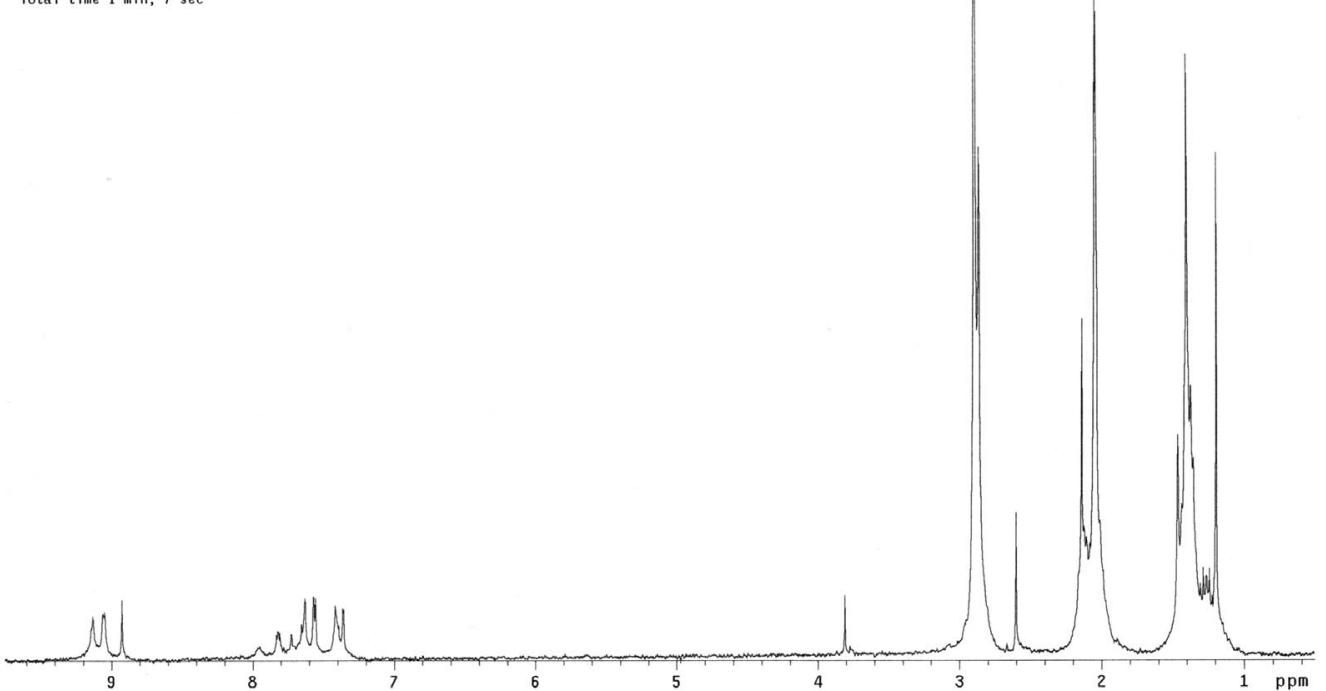




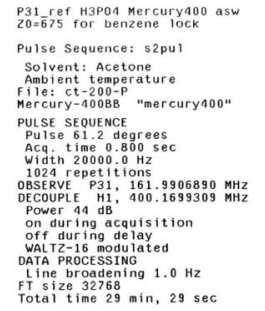

Compoun 15, P-31 spectrum

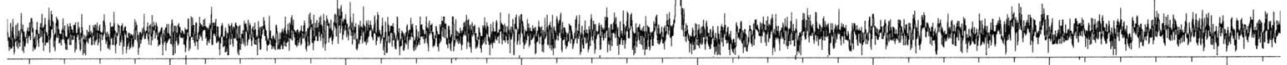

15

10

0

$-5$
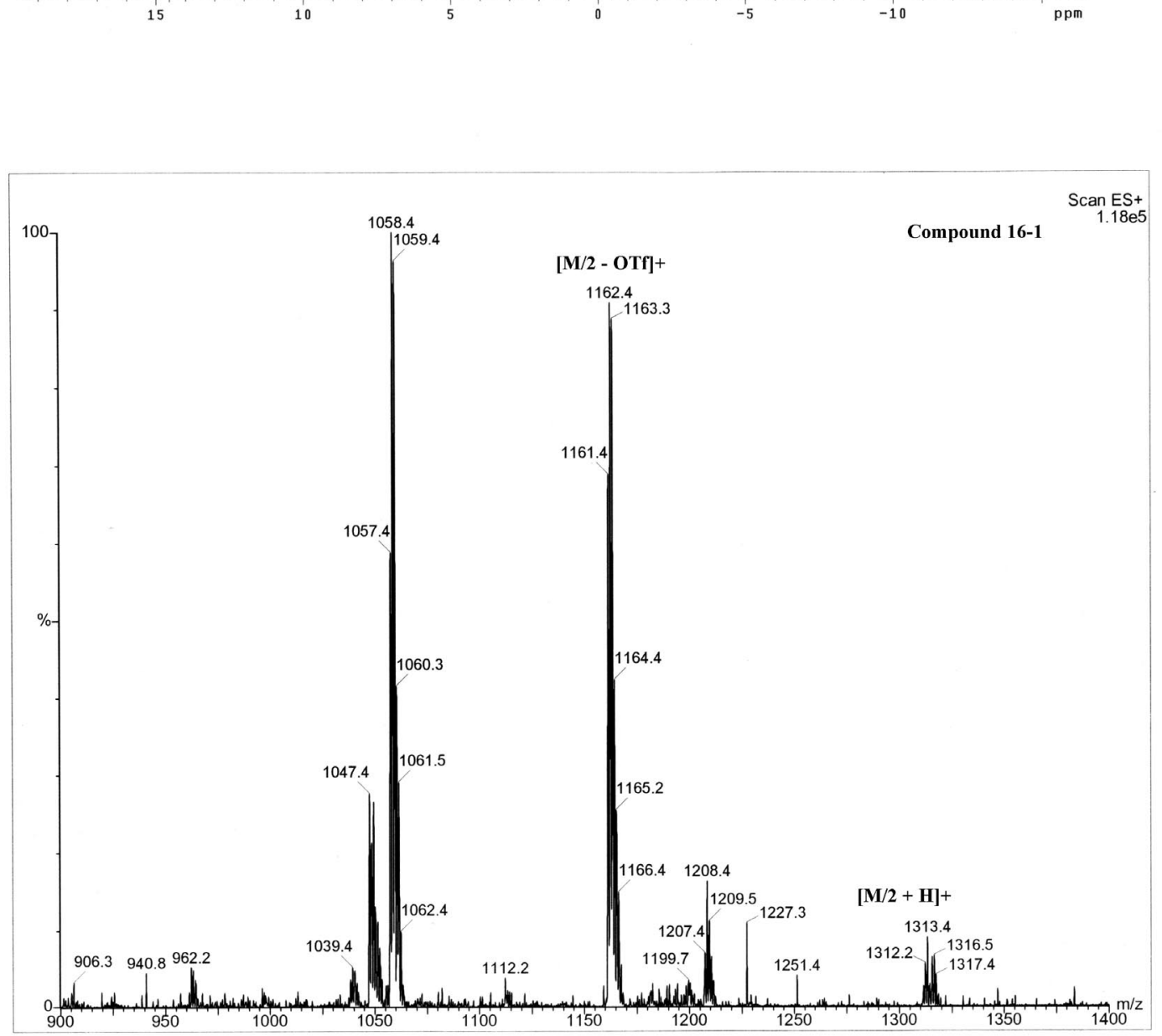


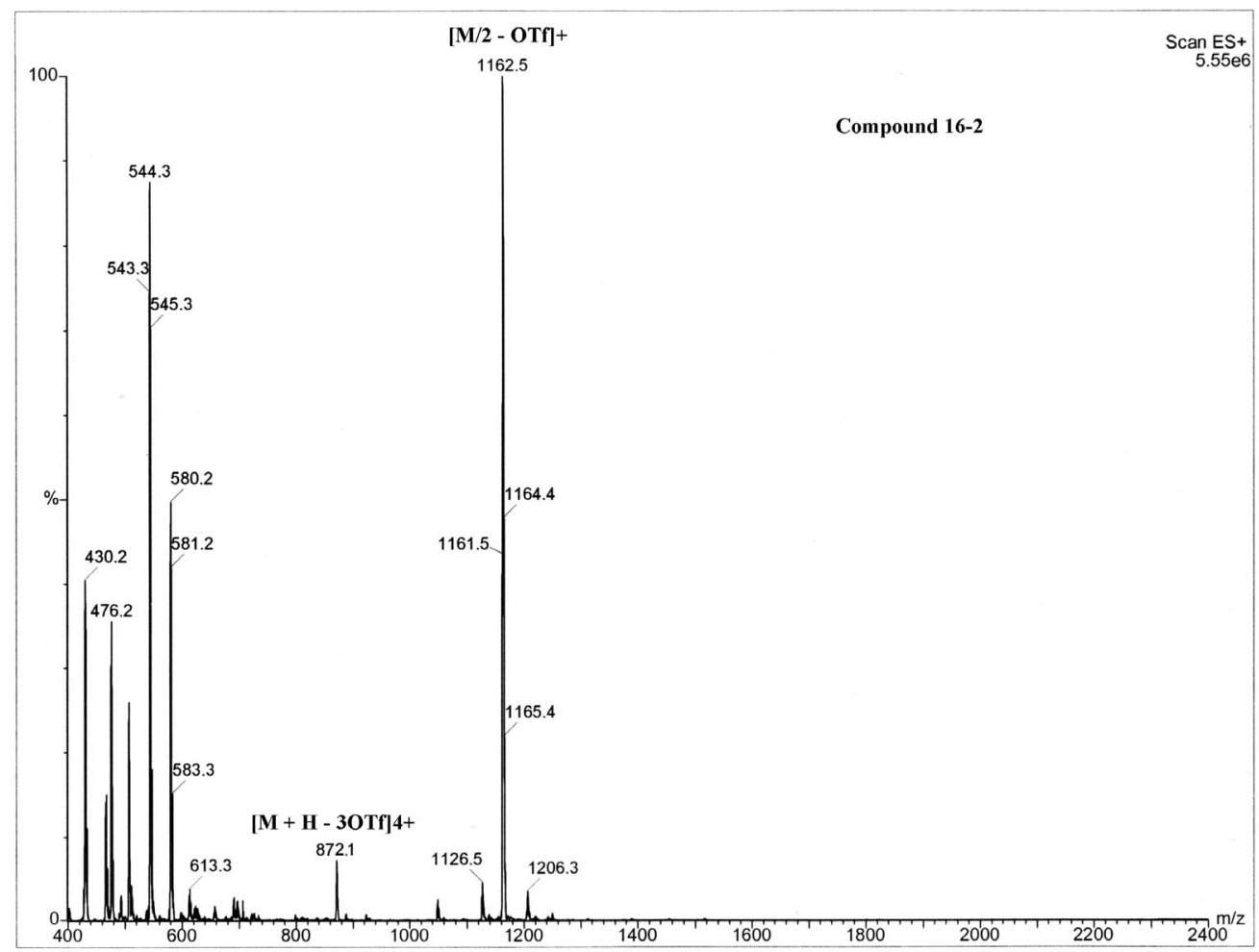

F19 OBSERYE
STANDARD PARAMETERS

Pulse Sequence: s2pul

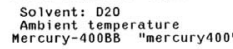

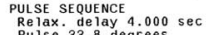

pulse 33.8 degrees

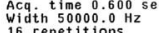

OBSERPE F19, $376.5434051 \mathrm{MHZ}$

OAIA PROCESSIMG
Dine broadening $0.3 \mathrm{~Hz}$
tine

Line broadening $0.3 \mathrm{~Hz}$
FT size

Compoun 16, F-19 spectrum

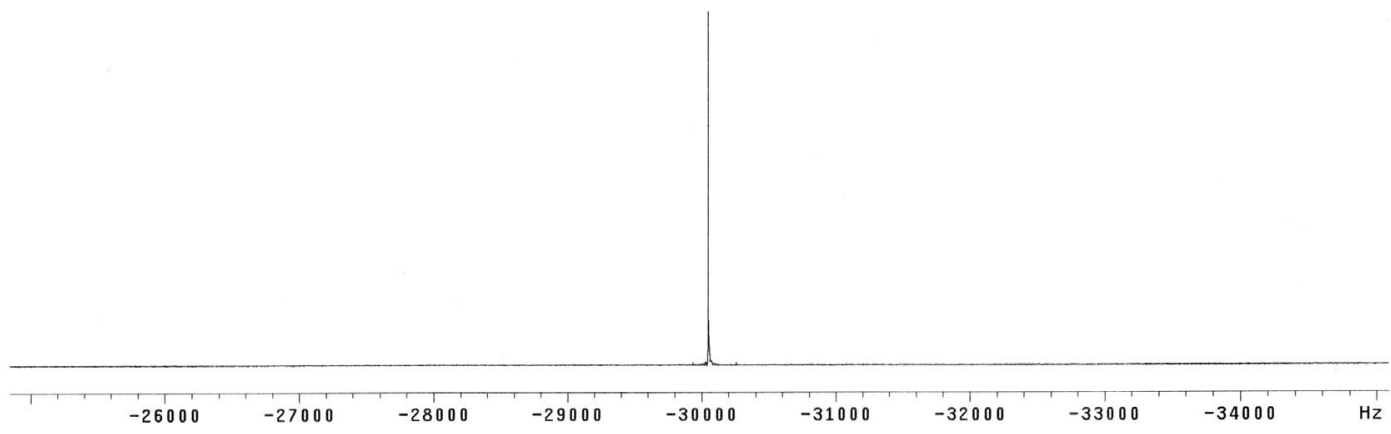




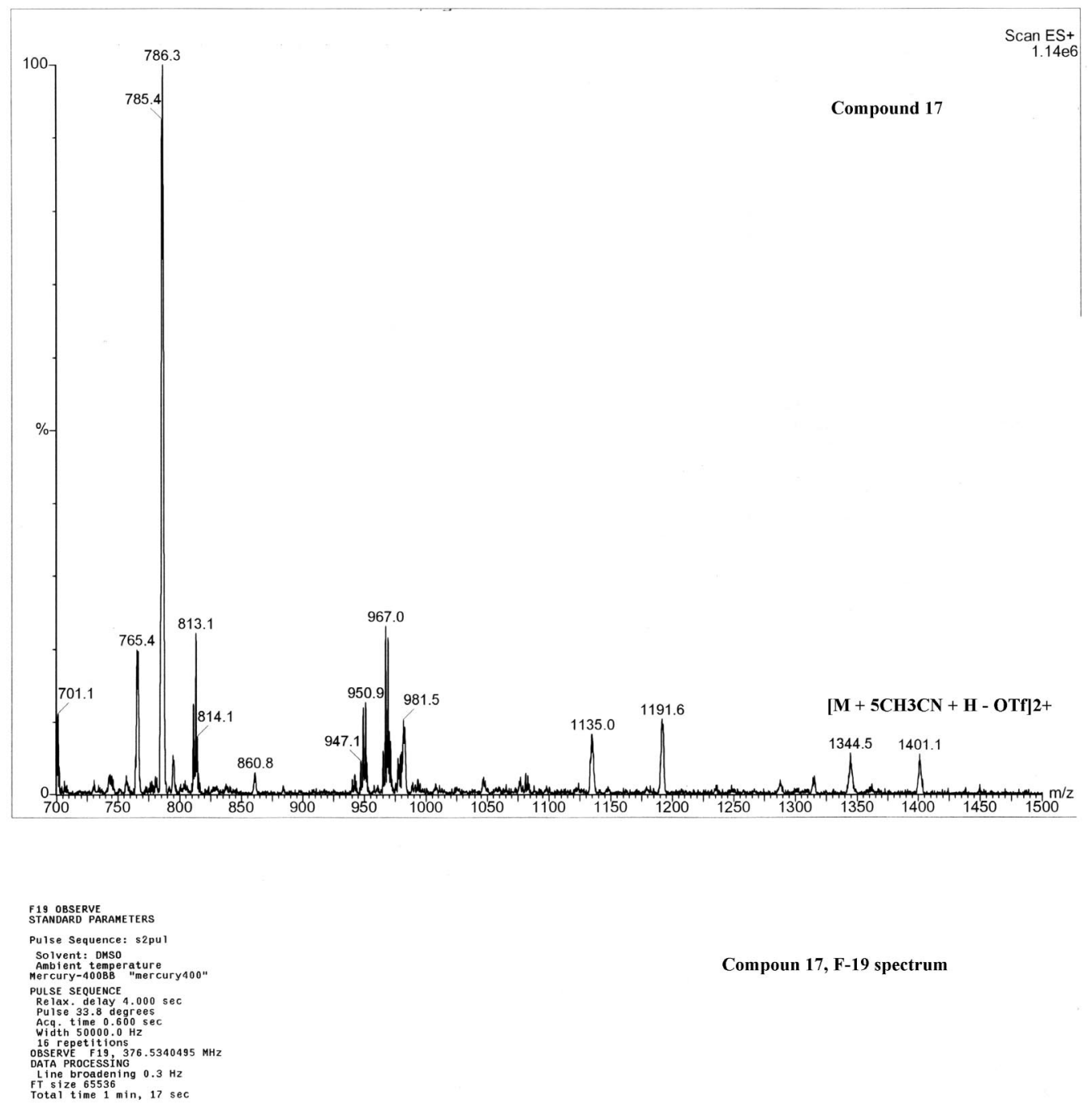

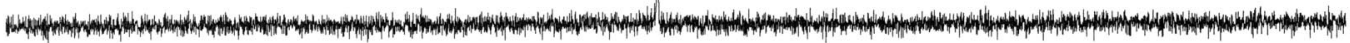



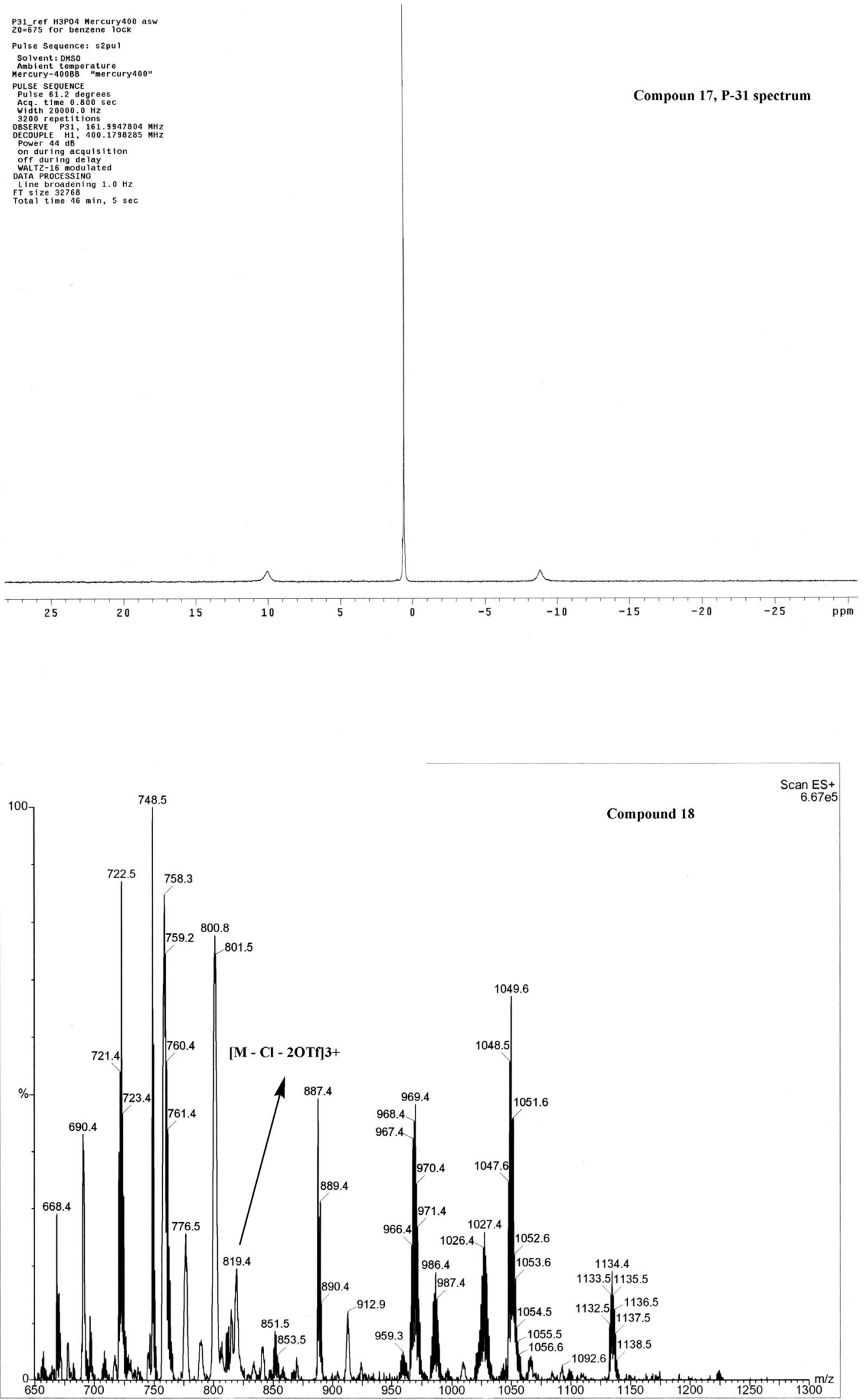